\title{
Association between CYP17A1, CYP19A1, and HSD17B1 Gene Polymorphisms in Hormone Synthesis pathway with Ovarian Cancer Risk
}

\author{
Gowtham Kumar $\mathrm{G}^{1}$, Andrea Francis ${ }^{1}$, Solomon Paul ${ }^{1}$, Chirag Molia ${ }^{1}$, Manickavasagam \\ $\mathrm{M}^{1}$, Usha Rani ${ }^{1}$, Ramya $\mathrm{R}^{1}$, and Nalini Ganesan ${ }^{1}$ \\ ${ }^{1}$ Sri Ramachandra Institute of Higher Education and Research
}

August 27, 2020

\begin{abstract}
Objective: To investigate the polymorphisms of genes in the steroidogenesis pathway to understand the etiological mechanisms to OC risk in the South Indian population Design: Case-Control Study Setting and Sample: Ovarian cancer cases (200) and healthy individuals (200) from the South Indian population. Methods: All the cases and controls were genotyped for SNPs by using allelic discrimination assay. Main outcome measures: Genetic distribution of SNPs of Steroidogenesis pathway genes in the South Indian population. Results: The observed results for rs743752, the homozygous CC genotype revealed significant association (OR; 1.68; 95\% CI, 1.25-2.26; $\mathrm{p}=<0.05)$ and the dominant model, recessive model and additive model showed a significant association with an OR of $1.62 ; 95 \% \mathrm{CI}, 1.09-2.42 ; \mathrm{p}=0.015$, OR of $0.29,95 \% \mathrm{CI}, 0.14-0.60 ; \mathrm{p}=<0.001$ and OR of $1.68,95 \% \mathrm{CI}, 1.25-2.26) ; \mathrm{p}=<0.001$ respectively in cases and controls for OC risk. In rs10046, the heterozygous CT genotype (OR; 1.61; 95\%CI $1.06-2.43 ; \mathrm{p}=0.023)$, the dominant $(\mathrm{OR} ; 1.65 ; 95 \% \mathrm{CI}, 1.11-2.45 ; \mathrm{p}=0.012)$ and the additive $(\mathrm{OR} ; 1.46 ; 95 \% \mathrm{CI}, 1.07-1.98 ; \mathrm{p}=0.015)$ models were found to be statistically significant. There was no significant association between rs605059 genotypes with ovarian cancer risk. Conclusions: To conclude, results indicated that the polymorphisms of CYP17A1 (rs743572) and CYP19A1 (rs10046) genes are associated with increased risk of ovarian cancer risk in South Indian population.
\end{abstract}

\section{Introduction}

Ovarian cancer $(\mathrm{OC})$ is the most lethal gynecological cancer accounts for $2.5 \%$ of all cancers and the fourth most common cancer and the seventh leading cause of deaths among women worldwide ${ }^{(1)}$. In India, OC is the third leading site of cancer in women trailing behind cervical and breast cancers ${ }^{(2,3)}$. A number of studies gave an account of several diagnostic markers in $\mathrm{OC}^{(4,5)}$. In general, $\mathrm{OC}$ is characterized based on the molecular-based markers, histopathological parameters and clinical factors include age, age at menarche, menopause status, and stage of the disease at diagnosis (FIGO).

The OC is an endocrine-related tumor that arises from the ovaries in which the hormones such as estrogen, progesterone and testosterone are synthesized ${ }^{(6)}$. These steroid hormones are synthesized by a group of enzymes called cytochrome $\mathrm{P} 450$ which regulates a variety of physiological and development progressions in humans ${ }^{(7)}$. This process starts with cholesterol, which synthesizes pregnenolone with the help of an enzyme $C Y P 11 A 1$. Furthermore, the pregnenolone and progesterone are converted into androstenedione by the enzyme $C Y P 17 A 1$. The final products estrogen, progesterone and testosterone were produced by the enzymes $C Y P 19 A 1$ and $H S D 17 B 1^{(8)}$. Ovarian steroidogenesis is a most complex process in which the granulosa and theca cells separate the enzymatic steps, which surround the oocyte and form a follicle ${ }^{(9)}$. In spite of the fact that the exact role of steroid hormones in OC risk is unclear ${ }^{(10,11)}$. 
The production of estrogens, progesterone and testosterone occurs through the steroidogenesis process where cholesterol is firstly synthesized into pregnenolone by the enzyme, CYP11A1. Thereafter, progesterone is produced from pregnenolone by HSD3B1 and HSD3B2. CYP17A1 converts pregnenolone and progesterone into androstenedione, a precursor for estrogen and testosterone production. The enzymes, CYP19A1, HSD17B1 and HSD17B2, are involved in the production of estrogens (estrone, estradiol, estriol) and testosterone.

Studies that evaluating both steroid hormone synthesis and its associated genes with OC risk are required to understand the etiological mechanism of hormones in ovarian carcinogenesis. An epidemiological study suggested that the genes in hormone biosynthesis pathway are the candidates because OC is an endocrinerelated tumor. The candidate genes CYP17A1, CYP19A1, and HSD17B1 with common allelic variants in the hormone biosynthesis pathway that may increase the estrogen concentration are likely to be considered as risk factors for breast cancer ${ }^{(6,12,13)}$. The genes CYP17A1,CYP19A1, and HSD17B1 are found to have an important role in the metabolism, synthesis, and maintaining androgen and estrogen hormone levels ${ }^{(14)}$. The polymorphisms in these genes may alter the level of estrogen and other hormones, which may increase the risk of OC. Due to the scarcity of studies that investigated the association of steroidogenesis gene polymorphisms with the OC risk. Hence, the current case-control study and was focused on the association between three major polymorphisms rs743572, rs10046, rs605059 of the steroid synthesis genes CYP17A1, CYP19A1, and $H S D 17 B 1$ respectively, in the South Indian population with Ovarian cancer risk.

\section{Material and Methods}

Sample Selection: In the present study, a total number of 400 individuals comprising 200 OC patients and 200 controls were recruited based on the specific inclusion and exclusion criteria after obtaining the informed consent. Blood samples of cases were collected from the patients with histological confirmation of OC, visited the department of oncology and gynecology at Sri Ramachandra Medical Centre, Chennai, India between 2015 and 2018. Meanwhile, age-matched controls were obtained from the 200 individuals who visited the outpatient department for the treatment of common ailments such as common cold, fever, etc., with no prior diagnosis of benign ovarian diseases; no history of hysterectomy, mastectomy or oophorectomy; no relatives with breast, ovarian, endometrial cancer; no physical or mental disability which would preclude their participation in the study. The study was approved by the Institutional Ethics Committee (IEC) of Sri Ramachandra University, Chennai, to collect blood samples from the study participants.

DNA Extraction: 3-5ml of peripheral blood samples from all the study participants were collected using EDTA containers (BD, NJ, USA). Genomic DNA was isolated from $200 \mu l$ of whole blood using a QIAamp DNA extraction kit by the manufacturer's protocol (Qiagen, Hilden, Germany. The isolated DNA samples were checked for quality and quantity using $0.8 \%$ agarose gel electrophoresis (AGE) and NanoDrop spectrophotometer (Thermo Fisher Scientific/NanoDrop Products, Wilmington, Delaware, USA) respectively which is then stored at $-20^{\circ} \mathrm{C}$ until analysis.

Genotyping: Taqman allelic discrimination assay was performed using predesigned probes and primers for genotyping CYP17A1 rs743572 (C_2852784_30), CYP19A1 rs10046 (C_8234731_30), and HSD17B1 rs605059 (C_2350902_10) polymorphisms. All the reactions were carried out using 384-well arrays at $60^{\circ} \mathrm{C}$ annealing temperature on the 7900HT Fast Real-Time PCR System (Applied Biosystems, Foster City, CA, USA). Each well-contained 5ul of total reaction mix with universal Taqman genotyping master mix (Applied Biosystems, Foster City, CA, USA), probes (Applied Biosystems, Foster City, CA, USA) and 10ng/ul of DNA sample. Genotypes were called using the SDS v2.4 Allelic Discrimination sequence detection software (Applied Biosystems, Foster City, CA, USA). Genotyping success rates were [?] 99\% for each genotype for all subgroups.

\section{Statistical Analysis:}

All the statistical analysis was performed using statistical software SPSS v25 (IBM Corporation) and Epi info v7.2.2.6 (Center for Disease Control and Prevention, Atlanta, Georgia). Genotype and allele frequencies for all three polymorphisms in cases and controls were calculated and tested for Hardy-Weinberg equilibrium. Association analysis of all the polymorphisms with OC risk was performed using odds ratio (OR) with a 
95\% confidence interval (CI). The association between the genotypes of all three polymorphisms with clinical characteristics was evaluated using the chi-square test. Two-tailed $\mathrm{P}$-value $<0.05$ was considered statistically significant. Bonferroni type adjustment was carried out for the statistically significant genotypes to test for multiple corrections. SNPstats, an online tool was used to estimate haplotype frequencies that use the Markov Chain Monte Carlo method (Sole' et al., 2006).

Funding: This work was supported by the Department of Biotechnology, India (6242P43/RGCB/PMD/DBT/ANDR/2015).

\section{Results:}

Characteristics of the cases and controls:

The present case-control study comprises 200 OC cases and 200 healthy volunteers to match the cases were called up to investigate the association of CYP17A1 (rs743572), CYP19A1 (rs10046) andHSD17B1 (rs605056) gene polymorphisms and its association with OC risk in South Indian population. Both cases and controls were selected based on the eligibility standards. The cases and controls were matched by age $(\mathrm{P}=0.996)$. Student's t-test revealed a significant association in the BMI $(\mathrm{p}=0.0002)$ and the significant difference was observed in menopausal status by using the chi-square test $(\mathrm{p}=0.008)$. There was no significant association found in menarche age. The demographic details of the cases and healthy individuals are presented in table 1.

Genotype frequency distribution among cases and controls:

The genotypic distribution of CYP17A1 (rs743572), CYP19A1(rs10046) and HSD17B1 (rs605056) gene polymorphisms in the control samples did not show any deviation from the Hardy-Weinberg equilibrium.

The genotype frequency distribution between OC patients and controls of all the studied polymorphism given in the table 2 and the distribution of alleles in genetic models were represented in table 3 . For the polymorphism rs743572 of CYP17A1 gene, our results revealed that the homozygous variant CC allele had 3.93 fold risk increased $\mathrm{OC}$ risk $(\mathrm{OR}=3.93 ; 95 \% \mathrm{CI}=1.86-8.28 ; \mathrm{P}-<0.001)$. Similarly the genetic models dominant, recessive and additive allele were found to be associated with a $1.62(\mathrm{OR}=1.62 ; 95 \%$ CI $=1.09-2.42 ; \mathrm{P}-0.0158), 0.29(\mathrm{OR}=0.29 ; 95 \% \mathrm{CI}=0.14-0.60 ; \mathrm{P}-<0.001)$ and $1.68(\mathrm{OR}=1.68 ; 95 \%$ $\mathrm{CI}=1.25-2.26 ; \mathrm{P}-<0.001)$ fold increased risk of OC respectively in South Indian population.

For the SNP rs10046 of the CYP19A1 gene, the heterozygous allele (CT) was at 1.61 folds (OR $=1.61$; $95 \% \mathrm{CI}=1.06-2.43)$ increased OC risk in patients when compared to controls. Additionally, results on the genetic models showed that the dominant allele had $1.65(\mathrm{OR}=1.65 ; 95 \% \mathrm{CI}=1.11-2.45) ; \mathrm{P}-0.023)$ folds and additive allele had 1.46 folds $(\mathrm{OR}=1.46 ; 95 \% \mathrm{CI}=1.07-1.98)$ increased in OC patients compared to controls. Furthermore, in cases of rs605056 polymorphism of $H S D 17 B 1$, no significant differences were seen in any of the genetic models between cases and controls with OC risk.

Haplotype analysis:

Haplotype analysis of the three polymorphisms revealed eight different haplotype combinations were observed in both cases and controls (Table 4). The results showed CCA and CTA were found to be higher in cases (0.2 and 0.07) compared to controls (0.13 and 0.04) with an OR of $0.46(0.27-0.79)$ and $0.40(0.18-0.91)$, respectively indicating that the possible contribution of the haplotypes to the risk of OC.

\section{Discussion:}

\section{Main Findings:}

To the best of our knowledge, this is the first study to provide solid evidence that the CYP17A1 (rs743572) and CYP19A1 (rs10046) polymorphisms showed significant association with ovarian cancer in South Indian population. However, the polymorphism rs605056 of HSD17B1 did not show any significant association with ovarian cancer in the same population. The haplotype analysis results showed that the combination alleles are contributed to the risk of OC. 


\section{Strength and limitations :}

In the present study, 400 subjects that include 200 clinical proven OC cases and 200 healthy volunteers as a control group were analyzed for the genotype of three polymorphisms rs743572 of CYP17A1, rs10046 of CYP19A1 and rs605056 of HSD17B1 genes. However, some limitations need to be considered in interpreting our findings. Firstly, the main considered limitation was the sample size due to the low incidence of OC in the Indian population especially South Indian. Further studies are needed to confirm the association of gene polymorphisms with OC risk. Although, our study revealed that polymorphisms rs743572 and rs10046 are significantly associated with $\mathrm{OC}$ in the Indian population. These selected genes are highly involved in the synthesis of steroid hormones (estrogen, androgen and progesterone) which is having importance in the progression of breast and $\mathrm{OC}^{(15)}$. The function of these hormone synthesis genes, which may affect the estrogen levels and its harmful metabolites, which prompts $\mathrm{OC}^{(16)}$. Due to the lack of studies on steroid hormone synthesis with OC risk, we had to compare our results for all the polymorphisms with those of similar studies on OC risk.

\section{Interpretation:}

The CYP17A1 is considered a key enzyme in the estrogen-signaling pathway. This gene was found to catalyze two different steps in the pathway, the 17, 20 lyases and 17 alpha hydroxylases reactions and it has an important function in synthesis, metabolism and maintaining the levels of estrogen and androgen hormones ${ }^{(17)}$. The polymorphism in the 5' promoter region has $\mathrm{T} / \mathrm{C}$ substitution that creates an additional SpI type (CCACC) promoter site. This could alter the recognition site for the MspAI recognition site ${ }^{(18)}$. This rs743572 polymorphism is considered an increased risk of various cancers ${ }^{(19-23)}$. A study showed that the rs743572 T/C polymorphism in the CYP17A1 gene may alter the binding characteristics of the promoter region and modifies the gene's function ${ }^{(18)}$. This could lead to a change in estrogen levels and the risk of OC. In the same way, our results were in agreement with many of the studies that reported the increased number of CC genotype than TT leads to increased circulating levels of estradiol ${ }^{(24,25)}$. A study, which was conducted in the North Indian population on breast cancer showed a significant association with CYP17A1 rs743572 polymorphism ${ }^{(26)}$. Furthermore, a study engaged by Konstantinos et al, confirms our results by suggesting that the $\mathrm{C}$ allele of rs743572 polymorphism might influence promoter activity with an increased amount of $C Y P 17 A 1$ mRNA transcription ${ }^{(27)}$. The rs743572 polymorphism increases the levels of androgen due to the induction of $C Y P 1 A 1$ gene expression that ultimately resulted in the development and progression of prostate disorders ${ }^{(28)}$.

CYP19A1 gene codes for the enzyme aromatase which plays a key role in the aromatization in which the androgens get converted into estrogens ${ }^{(29)}$. This $30 \mathrm{~kb}$ coding region and $93 \mathrm{~kb}$ regulatory gene is located on the chromosome $15 \mathrm{q} 21.2^{(30)}$. In the meantime, variations in this gene have the ability to fluctuate the levels of circulating hormones which allude to speculate that the polymorphisms such as rs10046, a T/C substitution at the 3' untranslated region of CYP19A1 gene, are having an impact on breast cancer risk $^{(31)}$. The exact function of this polymorphism is properly unknown, yet it has been claimed that this may lead to influence levels of mRNA and its stability ${ }^{(32)}$, therefore it can be associated with altered levels of the aromatase enzyme and affected estrogen metabolism. This rs10046 polymorphism was examined extensively in different populations. A study conducted on Chinese and a meta-analysis conducted in 20,098 subjects from Spain revealed an association between rs10046 and breast cancer risk ${ }^{(33,34)}$. However, no

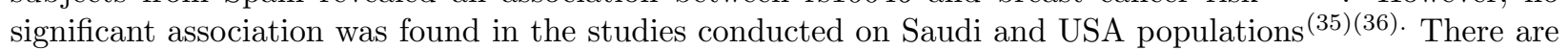
no studies available with respect to the association with OC. Since the CYP19A1 was found to be significantly associated with breast cancer risk by several studies. These inconsistent results in different populations in different studies may attribute to several risk factors and it is necessary to examine the association of those individual populations. In the present study, the heterozygous, dominant and $T$ allele was found to be higher in OC cases compared to controls and it is significantly associated with OC risk. These results suggest that the rs10046 polymorphism may have an impact on the progression of ovarian cancer. However, the rs605059 polymorphism of the HSD17B1 gene did not show a significant association with OC in any of the genetic models on the South Indian population. 
In the haplotype analysis, the haplotype distribution of the polymorphisms rs743572, rs10046 and rs605056 on OC had shown 8 different combinations of haplotypes in which 2 combinations (CCA and CTA) were found to be significantly higher in cases compared to controls, thus it reveals an association with OC risk. Furthermore, this is the first study to report the association of CYP17A1, CYP19A1, and HSD17B1 haplotypes with OC.

\section{Conclusion:}

In conclusion, our data provided the evidence for an association between the rs743572, rs10046 polymorphisms of genes CYP17A1 and CYP19A1 and OC risk, while no association was found with rs605059 polymorphism of the HSD17B1 gene.

Disclosure of Interests: The authors did not have any conflict of interest to declare. There are no financial, personal, political, intellectual or religious interests to disclose.

Contribution to Authorship: All authors (GGK, AMF, SFDP, CM, MM, UR, RR, NG) contributed to the design, participant recruitment, data collection, and conception in the present study. GGK, AMF was involved in the manuscript drafting and data analysis. GGK and CM were collected the data. All the other authors were involved in interpreting data and critically reviewing the manuscript. All the author has given the approval of the final version of the manuscript.

Details of Ethics Approval: The study was approved on 06.10 .2017 by the ethics committee of Sri Ramachandra Institute of Higher Education and Research (Reference Number - IEC-NI/17/JUN/60/80).

Funding: This work was supported by the Department of Biotechnology, India (6242P43/RGCB/PMD/DBT/ANDR/2015).

Data sharing and Data Accessibility: All the data that supports the findings of this study are included.

\section{Acknowledgment:}

We are grateful to the Department of Biotechnology (DBT), India for the funding. All the authors would like to thank all the study participants for volunteering to be involved in this study. Also, we are thankful to Dr. Mohana Priya from CRF, SRIHER for providing RT-PCR facilities to conduct the study. We are also grateful to the Department of Oncology and Department of Obstetrics and Gynecology, SRIHER for providing samples and the data.

\section{References :}

1. Desai A. Epithelial ovarian cancer: An overview. World J Transl Med. 2014 Apr;3(1):1.

2. Basu P, De P, Mandal S, Ray K, Biswas J. Study of 'patterns of care' of ovarian cancer patients in a specialized cancer institute in Kolkata, eastern India. Indian J Cancer. 2009;46(1):28-33.

3. Jana D, Mandal S, Mukhopadhyay M, Mitra D, Mukhopadhyay SK, Sarkar DK. Prognostic significance of HER-2/neu and survival of breast cancer patients attending a specialized breast clinic in Kolkata, Eastern India. Asian Pacific J Cancer Prev. 2012;13(8):3851-5.

4. Vollan HKM, Rueda OM, Chin SF, Curtis C, Turashvili G, Shah SP, et al. A tumor DNA complex aberration index is an independent predictor of survival in breast and ovarian cancer. Mol Oncol. 2015 Jan;9(1):115-27.

5. Chen M, Jin Y, Bi Y, Li Y, Shan Y, Pan L. Prognostic significance of lymphovascular space invasion in epithelial ovarian cancer. J Cancer. 2015;6(5):412-9.

6. Parazzini F, Franceschi S, La Vecchia C, Fasoli M. The epidemiology of ovarian cancer. Gynecol Oncol. 1991 Oct;43(1):9-23.

7. Miller WL, Auchus RJ. The molecular biology, biochemistry, and physiology of human steroidogenesis and its disorders. Endocr Rev. 2011 Feb;32(1):81-151. 
8. Ashton KA, Proietto A, Otton G, Symonds I, McEvoy M, Attia J, et al. Polymorphisms in genes of the steroid hormone biosynthesis and metabolism pathways and endometrial cancer risk. Cancer Epidemiol. 2010;34(3):328-37.

9. Voutilainen R, Tapanainen J, Chung BC, Matteson KJ, Miller WL. Hormonal regulation of P450scc (20,22-desmolase) and P450cl7 (17 $\alpha$-hydroxylase/17,20-lyase) in cultured human granulosa cells. J Clin Endocrinol Metab. 1986 Jul;63(1):202-7.

10. Claus EB, Schildkraut JM, Thompson WD, Risch NJ. The genetic attributable risk of breast and ovarian cancer. Cancer. 1996;77(11):2318-24.

11. Lukanova A, Kaaks R. Endogenous hormones and ovarian cancer: Epidemiology and current hypotheses. Cancer Epidemiol Biomarkers Prev. 2005;14(1):98-107.

12. Elmasry K, Gayther S. Epidemiology of ovarian cancer. In: Cancer of the Ovary. Academic Press; 2006. p. $1-19$.

13. Silva SN, Cabral MN, De Castro GB, Pires M, Azevedo AP, Manita I, et al. Breast cancer risk and polymorphisms in genes involved in metabolism of estrogens (CYP17, HSD17ß1, COMT and MnSOD): Possible protective role of MnSOD gene polymorphism Val/Ala and Ala/Ala in women that never breast fed. Oncol Rep. 2006;16(4):781-8.

14. Bugano DDG, Conforti-Froes N, Yamaguchi NH, Baracat EC. Genetic polymorphisms, the metabolism of estrogens and breast cancer: A review. Eur J Gynaecol Oncol. 2008;29(4):313-20.

15. Dumas I, Diorio C. Estrogen pathway polymorphisms and mammographic density. Anticancer Res. 2011 Dec;31(12):4369-86.

16. Garner EIO, Stokes EE, Berkowitz RS, Mok SC, Cramer DW. Polymorphisms of the estrogenmetabolizing genes CYP17 and catechol-O-methyltransferase and risk of epithelial ovarian cancer. Cancer Res. 2002 Jun;62(11):3058-62.

17. Eccles DM, Pichert G. Familial non-BRCA1/BRCA2-associated breast cancer. Vol. 6, Lancet Oncology. Lancet Oncol; 2005. p. 705-11.

18. Carey AH, Waterworth D, Patel K, White D, Little J, Novelli P, et al. Polycystic ovaries and premature male pattern baldness are associated with one allele of the steroid metabolism gene CYP17. Hum Mol Genet. 1994 Oct;3(10):1873-6.

19. Manolio TA. Genomewide association studies and assessment of the risk of disease. Feero WG, Guttmacher AE, editors. N Engl J Med. 2010 Jul 8 [cited 2020 Mar 19];363(2):166-76.

20. Chakraborty A, Murthy NS, Chintamani C, Bhatnagar D, Mohil RS, Sharma PC, et al. CYP17 gene polymorphism and its association with high-risk north Indian breast cancer patients. J Hum Genet. 2007 Feb 18;52(2):159-65.

21. Surekha D, Sailaja K, Rao DN, Padma T, Raghunadharao D, Vishnupriya S. Association of a CYP17 gene polymorphism with development of breast cancer in India. Asian Pacific J Cancer Prev. 2010;11(6):1653-7.

22. Hou L, Xu J, Gao YT, Rashid A, Zheng SL, Sakoda LC, et al. CYP17 MspA1 polymorphism and risk of biliary tract cancers and gallstones: A population-based study in Shanghai, China. Int J Cancer. 2006 Jun 1;118(11):2847-53.

23. Yazici H, Tigli H, Kadehci Z, Kucucuk S, Saip P, Issever H, et al. Are CYP17 genotypes a biomarker for ovarian cancer in patients with cancer history in their family? Oncol Res. 2006;16(1):43-7.

24. Miyoshi Y, Iwao K, Ikeda N, Egawa C, Noguchi S. Genetic polymorphism in CYP17 and breast cancer risk in Japanese women. Eur J Cancer. 2000;36(18):2375-9. 
25. Feigelson HS, Shames LS, Pike MC, Coetzee GA, Stanczyk FZ, Henderson BE. Cytochrome P450c17 $\alpha$ gene (CYP17) polymorphism is associated with serum estrogen and progesterone concentrations. Cancer Res. 1998 Feb 15;58(4):585-7.

26. Chattopadhyay S, Siddiqui S, Akhtar MS, Najm MZ, Deo SVS, Shukla NK, et al. Genetic polymorphisms of ESR1, ESR2, CYP17A1, and CYP19A1 and the risk of breast cancer: A case control study from North India. Tumor Biol. 2014 Jan 16;35(5):4517-27.

27. Agiannitopoulos K, Kyparissi A, Manginas A, Papamenzelopoulos S, Lamnissou K. Genetic variant in the CYP17 gene and risk of premature coronary artery disease. Hell J Cardiol. 2014;55(2):126-31.

28. Song J, Tao ZH, Liu XY, Gong S, Gan L. Relationship between CYP17 gene polymorphisms and risk of prostate cancer. Genet Mol Res. 2016 Feb 26;15(1).

29. Toda K, Terashima M, Kawamoto T, Sumimoto H, Yokoyama Y, Kuribayashi I, et al. Structural and functional characterization of human aromatase P-450 gene. Eur J Biochem. 1990 Oct 1 [cited 2020 Mar 19];193(2):559-65.

30. Bulun SE, Sebastian S, Takayama K, Suzuki T, Sasano H, Shozu M. The human CYP19 (aromatase P450) gene: Update on physiologic roles and genomic organization of promoters. In: Journal of Steroid Biochemistry and Molecular Biology. Elsevier Ltd; 2003. p. 219-24.

31. Dunning AM, Dowsett M, Healey CS, Tee L, Luben RN, Folkerd E, et al. Polymorphisms associated with circulating sex hormone levels in postmenopausal women. J Natl Cancer Inst. 2004 Jun;96(12):936-45.

32. Bampali K, Grassos C, Mouzarou A, Liakos C, Mertzanos G, Lamnissou K, et al. Genetic variant in the CYP19A1 gene associated with coronary artery disease. Durocher F, editor. Genet Res Int. 2015;2015:820323.

33. Zhang L, Gu L, Qian B, Hao X, Zhang W, Wei Q, et al. Association of genetic polymorphisms of ER- $\alpha$ and the estradiol-synthesizing enzyme genes CYP17 and CYP19 with breast cancer risk in Chinese women. Breast Cancer Res Treat. 2009;114(2):327-38.

34. Pineda B, García-Pérez MÁ, Cano A, Lluch A, Eroles P. Associations between Aromatase CYP19 rs10046 Polymorphism and Breast Cancer Risk: From a Case-Control to a Meta-Analysis of 20,098 Subjects. PLoS One. 2013;8(1):1-9.

35. Haiman CA, Hankinson SE, Spiegelman D, Brown M, Hunter DJ. No association between a single nucleotide polymorphism in CYP19 and breast cancer risk. Cancer Epidemiol Biomarkers Prev. 2002 Feb;11(2):2156 .

36. Alanazi M, Alabdulkarim HA, Shaik JP, Al Naeem AA, Elrobh M, Al Amri AA, et al. No associations between aromatase gene polymorphisms and breast cancer risk in Saudi patients. Onco Targets Ther. 2015 Sep 3;8:2453-9.

\section{Hosted file}

Tables and Legends_Association between CYP17A1, CYP19A1, and HSD17B1 Gene Polymorphisms in Hormone Synt available at https://authorea.com/users/353284/articles/477249-association-between-cyp17a1cyp19a1-and-hsd17b1-gene-polymorphisms-in-hormone-synthesis-pathway-with-ovarian-cancerrisk 